\title{
Critical-sized cartilage defects in the equine carpus
}

\section{Salonius, Eve}

2019-03-04

Salonius , E , Rieppo , L , Nissi , M J , Pulkkinen , H J , Brommer , H , Bruenott , A , Silvast , T S , Van Weeren , P R, Muhonen, V , Brama , P A J \& Kiviranta , I 2019 , ' Critical-sized cartilage defects in the equine carpus ', Connective Tissue Research , vol. 60 , no. 2 , pp. 95-106 . https://doi.org/10.1080/03008207.2018.1455670

http://hdl.handle.net/10138/312138

https://doi.org/10.1080/03008207.2018.1455670

unspecified

acceptedVersion

Downloaded from Helda, University of Helsinki institutional repository.

This is an electronic reprint of the original article.

This reprint may differ from the original in pagination and typographic detail.

Please cite the original version. 
3 Eve Salonius ${ }^{1 *}$, Lassi Rieppo ${ }^{2,3}$, Mikko J. Nissi ${ }^{4}$, Hertta J. Pulkkinen ${ }^{5}$, Harold Brommer ${ }^{6}$,

4 Anne Brünott ${ }^{6}$, Tuomo S. Silvast ${ }^{7}$, P. René van Weeren ${ }^{6}$, Virpi Muhonen ${ }^{1}$, Pieter A. J.

$5 \quad$ Brama $^{8}$, Ilkka Kiviranta ${ }^{1,9}$

6

71 Department of Orthopaedics and Traumatology, University of Helsinki, Helsinki, Finland

82 Research Unit of Medical Imaging, Physics and Technology, University of Oulu, Oulu,

$9 \quad$ Finland

103 Medical Research Center, University of Oulu and Oulu University Hospital, Oulu, Finland

114 Department of Applied Physics, University of Eastern Finland, Kuopio, Finland

125 Institute of Biomedicine, University of Eastern Finland, Kuopio, Finland

136 Department of Equine Sciences, Utrecht University, Utrecht, The Netherlands

147 SIB Labs, University of Eastern Finland, Kuopio, Finland.

158 Section of Veterinary Clinical Sciences, School of Veterinary Medicine, University College

16 Dublin, Ireland

179 Department of Orthopaedics and Traumatology, Helsinki University Hospital, Helsinki,

18 Finland 
20 *Address correspondence to: Eve Salonius, University of Helsinki, Department of Surgery,

21 Biomedicum Helsinki, Haartmaninkatu 8, 00290, Helsinki, Finland. Tel. +358-9-471-71919,

22 eve.salonius@helsinki.fi

23 E-mail addresses: eve.salonius@ helsinki.fi (E. Salonius), lassi.rieppo@oulu.fi (L. Rieppo), 24 mikko.nissi@uef.fi (M. Nissi), hertta.pulkkinen@live.com (H.J. Pulkkinen),

25 H.Brommer@uu.nl (H. Brommer), anne@ brunott.biz (A. Brünott), tuomosilvast@gmail.com

26 (T.S. Silvast), r.vanweeren@uu.nl (P.R. van Weeren), virpi.muhonen@ helsinki.fi (V.

27 Muhonen), pieter.brama@ucd.ie (P.A.J. Brama), ilkka.kiviranta@ helsinki.fi (I. Kiviranta)

28

29 Running title: Critical-sized cartilage defects in the equine carpus

30

31 Funding declaration: This study was funded by the Academy of Finland (Grant \#285909)

32 and the Finnish Funding Agency for Innovation Tekes (Grant 3344/31/03). The funding

33 sources had no role in the study design, collection, analysis and interpretation of data; in the

34 writing of the manuscript; and in the decision to submit the manuscript for publication. 
Aim: The horse joint, due to its similarity with the human joint, is the ultimate model for translational articular cartilage repair studies. This study was designed to determine the critical size of cartilage defects in the equine carpus and serve as a benchmark for the evaluation of new cartilage treatment options.

Materials and Methods: Circular full-thickness cartilage defects with a diameter of 2, 4 and 8 $\mathrm{mm}$ were created in the left middle carpal joint and similar osteochondral (3.5 $\mathrm{mm}$ in depth) defects in the right middle carpal joint of five horses. Spontaneously formed repair tissue was examined macroscopically, with MR and $\mu \mathrm{CT}$ imaging, polarized light microscopy, standard histology and immunohistochemistry at 12 months.

Results: Filling of $2 \mathrm{~mm}$ chondral defects was good $(77.8 \pm 8.5 \%)$ but proteoglycan depletion was evident in Safranin-O staining and gadolinium-enhanced MRI $\left(T_{1 G d}\right)$. Larger chondral defects showed poor filling $(50.6 \pm 2.7 \%$ in $4 \mathrm{~mm}$ and $31.9 \pm 7.3 \%$ in $8 \mathrm{~mm}$ defects). Lesion filling in 2, 4 and $8 \mathrm{~mm}$ osteochondral defects was $82.3 \pm 3.0 \%, 68.0 \pm 4.6 \%$ and $70.8 \pm 15.4 \%$, respectively. Type II collagen staining was seen in $9 / 15$ osteochondral defects but only in

$51 \quad 1 / 15$ chondral defects. Subchondral bone pathologies were evident in 14/15 osteochondral 52 samples but only in 5/15 chondral samples. Although osteochondral lesions showed better 53 neotissue quality than chondral lesions, the overall repair was deemed unsatisfactory because 54 of the subchondral bone pathologies.

55 Conclusions: We recommend classifying $4 \mathrm{~mm}$ as critical osteochondral lesion size and $2 \mathrm{~mm}$ 56 as critical chondral lesion size for cartilage repair research in the equine carpal joint model. 
57 Keywords: cartilage repair; animal model; spontaneous repair; preclinical research; critical58 sized defect 


\section{Introduction}

60 Animal models are used for the evaluation of the efficacy of new surgical techniques. When

61 investigating articular cartilage repair in vivo, joint size and cartilage thickness are considered

62 key factors in defining the most appropriate species. $(1,2)$ The joint size, cartilage thickness

63 and gait mechanics of the horse are closest to those of humans. $(3,4)$ Moreover, naturally

64 occurring equine cartilage lesions have similar etiology as human lesions. $(1,3,4)$ These

65 similarities allow for a realistic evaluation of novel methods for cartilage repair. In the equine

66 model, stifle, tarsotibial and carpal joints have been used in translational cartilage repair

67 research. To enable effective use of the equine model in translational cartilage research, the

68 intrinsic repair capacity of equine cartilage in the specific joint must be known.

69 A critical-sized lesion is a lesion of a size beyond which the defect does not heal

70 spontaneously. Knowledge about critical lesion sizes in animal experiments is necessary for

71 cost reduction and minimizing the suffering of animals while still providing reliable data on

72 the effect of the studied technique. Critical lesion size used in previous equine studies has

73 been defined as lesion size beyond which any void made is not filled. (5) However, tissue

74 quality should also be taken into consideration when defining cartilage repair. Aiming at

75 tissue regeneration, i.e. restoration of normal tissue architecture and function, instead of

76 merely filling the defects is paramount for achieving durable results. (6) Therefore, this kind

77 of defect filling cannot be considered to be successful healing.

78 There are no recent studies on spontaneous cartilage repair in the equine carpus, and previous

79 studies have generally used basic methods, such as macroscopic inspection, standard

80 histology and basic biochemistry for the assessment of repair tissue quantity and quality. $(5,7)$ 
81 Apart from this, to our knowledge, there are no data on the long-term evolution of artificially

82 made superficial chondral lesions in horses. Given the increasing recognition of the equine

83 model for the evaluation of cartilage repair techniques, $(1,3,8)$ and the equine carpus being the

84 most common site of naturally occurring osteoarthritis after metacarpophalangeal joint, (9)

85 our study focused on characterization of the long-term spontaneous repair of variably sized

86 chondral and osteochondral defects in the equine carpus using state-of-the-art analytical

87 techniques. As small cartilage defects have been thought to heal well, $(3,5,8)$ we hypothesized

88 that the critical defect size would be larger than $2 \mathrm{~mm}$ in diameter. The information obtained

89 in this study can be used as a benchmark when evaluating the effect of different techniques

90 aiming at cartilage regeneration in an equine translational model, as it defines to what extent

91 lesions of different sizes will heal spontaneously over a long period (12 months) in the equine

92 carpus.

94 Methods

95 Surgical procedure

96 Five 24-month-old horses (Equus caballus) were included in this study. The study was

97 authorized by the Utrecht University Animal Experiments Committee (0412.0601, Utrecht,

98 The Netherlands) in compliance with the Dutch Act on Animal Experimentation. The animal

99 care was in accordance with Utrecht University guidelines. Surgery was performed under

100 general anesthesia following routine clinical procedures. All the horses were assessed

101 clinically and radiologically prior to inclusion in the study and were found to be skeletally

102 mature and to present no abnormalities. 
103 The horses received meloxicam pre-operatively $\left(0.6 \mathrm{mg} / \mathrm{kg}, i . v .\right.$, Metacam ${ }^{\circ}$, Boehringer

104 Ingelheim). The middle carpal joints were approached through a lateral-dorsal and medio-

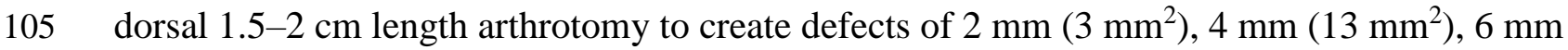

$106\left(28 \mathrm{~mm}^{2}\right)$ and $8 \mathrm{~mm}\left(50 \mathrm{~mm}^{2}\right)$ in diameter on the $2^{\text {nd }}, 3^{\text {rd }}$ and $4^{\text {th }}$ carpal bones as shown in

107 Figure 1. Defects were pre-punched with a 2, 4, 6 or $8 \mathrm{~mm}$ skin biopsy punch. For chondral

108 defects, cartilage was carefully removed with ring curettes onto the level of calcified cartilage

109 (approximately $1 \mathrm{~mm}$ in depth) in the left carpus. For osteochondral lesions created in the

110 right carpus, drilling was performed under continuous lavage with Ringer's solution using a

111 hand drill. A 2, 4, 6 or $8 \mathrm{~mm}$ pointed drill bit was initially used, followed by a custom-made

112 flattened drill bit of the same size and a custom-made drill sleeve to provide a uniform defect

113 with a flattened bottom and controlled depth of $3.5 \mathrm{~mm}$. Healthy cartilage adjacent to the

114 lesions served as control for all defects.

115 Post-operatively, the animals were confined to individual box-stalls $(3.5 \times 3.5 \mathrm{~m})$ for two

116 weeks, after which a gradual six-week rehabilitation program consisting of incremental

117 controlled walking started. Thereafter, depending on the season and weather conditions, the

118 animals were turned out to pasture or kept in box stalls with daily exercise of 20-30 minutes

119 in a mechanical horse walker. The exercise regimen was identical for all horses. Synovial

120 fluid and blood samples were collected at weeks 0, 2, 6, 14, 26, 38 and immediately after

121 euthanasia. The total follow-up period was 12 months during which the lesions were allowed

122 to heal spontaneously.

123 The $6 \mathrm{~mm}$ lesions created in os carpale $I V$ were used in other studies. (10-12) As their

124 processing was different from the other samples, the $6 \mathrm{~mm}$ lesions are not included in this

125 study. 
127 After sacrificing the animals, the carpal joints were opened and macroscopic photographs

128 were taken. Cylindrical osteochondral samples (14 $\mathrm{mm}$ in diameter and approximately $1 \mathrm{~cm}$

129 in depth) were taken using a hollow drill that was centered over the original lesion. The

130 samples were frozen and stored at $-20^{\circ} \mathrm{C}$ until further processing.

\section{$131 \quad$ Micro-computed tomography $(\boldsymbol{\mu C T})$}

132 The samples were thawed in PBS supplemented with inhibitors of metalloproteinases [5 $\mathrm{mM}$

133 ethylenediamine tetraacetic acid (EDTA) disodium salt (VWR International, Fontenay,

134 France) and $5 \mathrm{mM}$ benzamidine hydrochloride (Sigma-Aldrich, St. Louis, MO)], and

135 analyzed with a SkyScan-1172 scanner (SkyScan, Aartselaar, Belgium). The volume of

136 interest was a cylinder with the diameter of the defect size and height of $6 \mathrm{~mm}$. In control

137 samples, the diameter was $8 \mathrm{~mm}$. The data was analyzed for the structural bone parameters:

138 bone volume fraction $(B V / T V)$, trabecular thickness $(T b . T h)$, trabecular spacing $(T b . S p)$, and

139 trabecular number $(T b . N)$.

\section{Magnetic resonance imaging (MRI)}

141 Thawed samples were MR imaged with a 9.4 T device (Oxford 400 NMR vertical magnet;

142 Oxford Instruments, Witney, England), equipped with a Varian DirectDrive console (VnmrJ

143 2.3, Varian, Palo Alto, CA, USA) and a $19 \mathrm{~mm}$ quadrature volume coil (RAPID Biomedical,

144 Rimpar, Germany). The specimens were placed in a test tube and immersed in saline. $T_{2}$

145 relaxation time was measured in a single slice of $1 \mathrm{~mm}$ thickness using a single echo spin

146 echo sequence with TEs of 12, 24, 50, 80 and $110 \mathrm{~ms}$, a TR of $2.5 \mathrm{~s}$ and in-plane resolution of

$14770 \times 140 \mu \mathrm{m}$. Native $T_{1}$ relaxation time was measured in the same slice with the same 
148

149

150

151

152

153

154

155

156

157

158

159

160

161

162

163

164

165

166

167

168

169

resolution, using a progressive saturation recovery sequence with TRs of 0.3, 0.6, 1.2, 2.4 and $4.8 \mathrm{~s}$ and TE of $11.7 \mathrm{~ms}$. After the first scans, the specimens were immersed in a $1.0 \mathrm{mM} \mathrm{Gd}-$

DTPA $^{2-}$ solution for 20 hours at room temperature (RT), after which $T_{1 \mathrm{Gd}}$ was measured using the same saturation recovery sequence with the same resolution, but with TRs of $0.1,0.2,0.4$, 0.8 and 1.6 s. Two regions of interest (ROIs) were defined in the MR images as exemplified in Figure 2: ROI 1 covered exclusively any repair tissue at the lesion sites of the samples, regardless of its location (repair tissue only). ROI 2 was spatially aligned with the surrounding healthy cartilage and located where the repair tissue assumingly should be if everything was perfectly healed, and further split into superficial and deep halves (upper and lower part of the cartilage). A control ROI was defined in the adjacent healthy tissue and also split into superficial and deep halves.

\section{Polarized light microscopy}

After the imaging studies, the samples were processed for histology. The sample cylinders were fixed in $10 \%$ formalin for 48 hours at RT. The samples were decalcified in $10 \%$ EDTA and $4 \%$ formaldehyde in $0.1 \mathrm{M}$ phosphate buffer at RT, cut in half, dehydrated in ascending alcohol series, and embedded in paraffin. Tissue sections of $5 \mu \mathrm{m}$ in thickness were cut from the middle of the lesion.

Unstained tissue sections were measured using polarized light microscopy (Leitz Ortholux II POL, Leitz Wezlar, Wezlar, Germany). (13) The repair tissue was evaluated using a 300- $\mu$ mwide ROI, which was divided into ten layers of equal thicknesses for the analysis. The orientation of collagen fibrils in relation to the cartilage surface (0-90 degrees), and parallelism index (PI), which describes the randomness of fibril orientations within the pixel 
$170 \quad(0-1$, where 0 indicates completely random organization and 1 indicates completely parallel

171 organization), (13) were determined from the most superficial, middle and the deepest

172 section.

173

174

175

176

177

178

179

180

181 lesions and $3.5 \mathrm{~mm}$ for osteochondral lesions. As the ROI for the osteochondral defects

182 extended into the subchondral bone, the natural trabecular spaces in the sections resulted in

183 empty spaces and thus in a smaller filling degree in the healthy osteochondral control samples

184 than in the chondral samples.

185 The Safranin-O stained tissue sections in study III were evaluated using the OARSI

186 histopathology score validated for equine cartilage, in which each parameter is evaluated 0-4

187 where 0 represents healthy cartilage tissue.(16) The sections were scored by three

188 independent, blinded observers and an average of the scores was used. The defects that lacked 189 any repair tissue were given the worst score of 4 for each parameter. 
190 A previously published protocol (14) was used to evaluate the staining for type I and type II

191 collagen. Briefly, the sections were digested with hyaluronidase ( $2 \mathrm{mg} / \mathrm{ml}$, Sigma-Aldrich)

192 and pronase $(2 \mathrm{mg} / \mathrm{ml}$, Calbiochem, Merck KGaA), and immersed in hydrogen peroxide

193 (EnVision®+ System-HRP (AEC), Dako North America Inc.) to block endogenous

194 peroxidase activity. Non-specific staining was blocked with 10\% normal goat serum (Dako

195 Denmark A/S, Glostrup, Denmark). The sections were then incubated overnight at $4^{\circ} \mathrm{C}$ with

196 primary antibodies against collagen type II (ab34712, Abcam) and collagen type I (ab34710),

197 and diluted to $4 \mu \mathrm{g} / \mathrm{ml}$ with PBS supplemented with $1 \%$ bovine serum albumin (Sigma-

198 Aldrich). Horseradish peroxidase (HRP)-conjugated goat anti-rabbit secondary antibody

199 (Dako) was then applied. Antibody binding was visualized with AEC substrate chromogen

200 (Dako). The staining of each sample was evaluated under light microscopy.

\section{$201 \quad$ Statistical analysis}

202 Confidence intervals and standard errors were calculated with the IBM SPSS Statistics 22

203 software. Osteochondral and chondral lesions of the same diameter were compared to each

204 other. The significances of differences in the $\mu \mathrm{CT}$, MRI and polarized light microscopy

205 parameters were evaluated with a pairwise $t$ test, and Sidak adjustment was made for multiple

206 testing. Significances of difference in lesion filling was calculated with permutation type

207 ANOVA testing and Sidak adjustment. Comparisons of lesions and control tissue were made

208 with permutation type ANOVA testing with Dunnet method. A $p$ value under 0.05 was used

209 as the threshold to indicate a statistically significant difference.

\section{Results}


213 The surgeries were uneventful and all animals recovered well. All the horses demonstrated a

214 pattern of decreasing joint effusion and lameness after surgery that can be expected during the

215 normal healing of an arthrotomy in clinical cases. Joint effusion and lameness were minimal

216 at postoperative day 10 during suture removal and all the horses were fully recovered by 3-4

217 weeks post-surgery. No clinical abnormalities were noticed in any of the horses during the

218 remainder of the experiment.

\section{Gross appearance of the repair tissue}

220 Most of the $2 \mathrm{~mm}$ lesions showed good macroscopic filling ( 5 of 5 osteochondral and 3 of 5

221 chondral defects) (Figure 3). The $4 \mathrm{~mm}$ lesions were clearly distinguishable from the

222 surrounding healthy cartilage and were incompletely filled. One chondral $4 \mathrm{~mm}$ lesion

223 (animal D) showed rather good filling. Each of the $8 \mathrm{~mm}$ lesions was incompletely filled. No

224 degenerative changes were detected.

225

\section{Micro-computed tomography}

Visually, the bone structure was normal under the chondral lesions of $2 \mathrm{~mm}$ and $4 \mathrm{~mm}$ in diameter (Figure 4). Bone compactness was visually observed to be decreased in 4 of the 5 chondral lesions with a diameter of $8 \mathrm{~mm}$, and one of these samples showed a small subchondral bone erosion. There were subchondral bone changes in all but one of the osteochondral defects (the exception being horse E, $2 \mathrm{~mm}$ lesion). Only two osteochondral samples with a diameter of $2 \mathrm{~mm}$ presented without cyst-like bone changes. All osteochondral lesions of $4 \mathrm{~mm}$ and $8 \mathrm{~mm}$ in diameter had unhealed bone or a cyst-like bone lesion. There were no clear trends in the numeral $\mu \mathrm{CT}$ data and the individual variation between the 
234 samples was large. No statistically significant differences were found between the chondral

235 and osteochondral defects but the trabeculae were thinner in osteochondral defects of all sizes

236 than in healthy control tissue $(p=0.003)$.

\section{Magnetic resonance imaging}

238 The relaxation times in ROI 1 (repair tissue only) showed no clear trends with respect to the

239 increasing lesion diameter (Figure 5a). However, $T_{1 \mathrm{Gd}}$ relaxation times were shorter $(p=0.014$

240 and $p<0.001$ for osteochondral and chondral lesions, respectively) and $T_{1}$ relaxation times

241 slightly longer ( $p=0.156$ and $p=0.037$ for osteochondral and chondral lesions, respectively) in

242 the repair tissues than in the control samples (Figure 5).

243 In all ROIs, the osteochondral lesions had longer $T_{1 \mathrm{Gd}}$ relaxation times than the chondral

244 lesions, indicative of a higher proteoglycan content. However, no statistically significant

245 differences were observed. In ROI 1, the $T_{2}$ relaxation time was shorter in the $8 \mathrm{~mm}$ wide

246 osteochondral defects than in the chondral defects $(33.8 \pm 0.8 \mathrm{~ms}$ for osteochondral and

$24741.0 \pm 1.3 \mathrm{~ms}$ for chondral lesions, $p=0.020$ ). ROI 2 (lesion area aligned to adjacent healthy

248 cartilage) showed increasing $T_{2} \mathrm{~S}$ with larger lesion diameters and towards the cartilage

249 surface. Osteochondral lesions with a diameter of $8 \mathrm{~mm}$ deviated from this trend and showed

250 lower $T_{2}$ values $(48.9 \pm 8.4 \mathrm{~ms})$ than the $4 \mathrm{~mm}$ lesions $(59.2 \pm 10.3 \mathrm{~ms})$. The $8 \mathrm{~mm}$ lesions also

251 demonstrated a significant difference between the osteochondral and chondral samples in the

252 deep part of ROI 2 (103.9 $\pm 12.2 \mathrm{~ms}$ for chondral and $48.9 \pm 8.4 \mathrm{~ms}$ for osteochondral defects,

253 respectively, $p=0.020)$.

254 The $T_{1}$ relaxation time of the chondral samples in ROI 2 showed a trend of increasing with

255 lesion diameter and from the deep part of the tissue towards the cartilage surface (Figure $5 \mathrm{~b}$ 
and c). The $T_{1}$ relaxation times of all chondral lesions were higher than those of the control

257 tissue $(p<0.001)$. The largest variation in $T_{1}$ relaxation time was noted for osteochondral

258 lesions with a diameter of $8 \mathrm{~mm} . T_{1}$ relaxation time did not show significant differences

259 between the groups in either of the ROIs.

260 Changes in the relaxation times were visually observed, especially in the $T_{1 G d}$ relaxation time

261 between the lesion sites and adjacent tissue, exemplified here in the cases of the $2 \mathrm{~mm}$ lesions

262 (Figure 2). While the relaxation times of the subchondral bone are not shown, also differences

263 in the MRI signal of the subchondral bone immediately below the lesion site were observed

264 between the lesion types: a uniform appearance of the signal is seen below the chondral

265 lesions, while an area of increased signal was present in the vicinity of the osteochondral

266 lesions, indicating an increased water content in the area (Figure 2).

\section{Polarized light microscopy}

268 Polarized light microscopy showed high parallelism indexes (PI) in all samples (Figure 6).

269 Chondral lesions with a diameter of $2 \mathrm{~mm}$ showed a higher parallelism of collagen fibrils than

270 osteochondral lesions in the deep part of the repair tissue $(0.891 \pm 0.02$ for chondral and

$2710.787 \pm 0.03$ for osteochondral lesions, $p=0.042$ ). Otherwise, no statistically significant

272 differences in the PI between the lesion diameters or the lesion depths were detected.

273 Collagen orientation showed large variations between the groups and between individual

274 samples. Collagen orientation changed toward the typical tangential orientation in the

275 superficial part of the $2 \mathrm{~mm}$ lesions (Figure 7a) and deviated from what was expected in the

276 larger lesions. The osteochondral samples showed a higher level of fibril organization than the

277 chondral samples in the deep part of the tissue, the collagen orientation being $61.6 \pm 4.3^{\circ}$ for 4 
$\mathrm{mm}$ and $69.5 \pm 2.7^{\circ}$ for $8 \mathrm{~mm}$ osteochondral defects, and $35.4 \pm 7.0^{\circ}$ for $4 \mathrm{~mm}$ and $33.6 \pm 2.2^{\circ}$ for $8 \mathrm{~mm}$ chondral defects ( $p=0.047$ and $p=0.004$ for $4 \mathrm{~mm}$ and $8 \mathrm{~mm}$ lesions, respectively).

\section{Histological repair quality}

Lesion filling was analyzed from the Safranin-O stained sections. Filling of the osteochondral control samples was $81.7 \pm 0.2 \%$ and filling of chondral controls was $99.4 \pm 4.7 \%$. Lesion filling was most complete $(82.3 \pm 3.0 \%)$ in the osteochondral lesions in which the repair tissue reached the level of the surrounding cartilage surface in all of the $2 \mathrm{~mm}$ lesions (Figure 7 , Figure 8). On the other hand, 4 of 5 of the $4 \mathrm{~mm}$ lesions and 1 of 5 of the $8 \mathrm{~mm}$ lesions presented with repair tissue non-aligned with the surrounding cartilage with filling of $68.0 \pm 4.6 \%$ for $4 \mathrm{~mm}$ osteochondral defects and $70.8 \pm 15.4 \%$ for $8 \mathrm{~mm}$ defects, respectively. All of the $2 \mathrm{~mm}$ chondral lesions showed good lesion filling $(77.8 \pm 8.5 \%)$ whereas filling of the $4 \mathrm{~mm}$ chondral defects was $50.6 \pm 2.7 \%$ and filling of the $8 \mathrm{~mm}$ defects was $31.9 \pm 7.3 \% .9$ of 10 of the $4 \mathrm{~mm}$ and $8 \mathrm{~mm}$ lesions showed only small islands of unstained repair tissue or even a complete absence of repair cartilage in the histological sections. Islands of repair tissue occurred at sites where the subchondral bone plate was disrupted (Figure 8). None of the defects showed lateral expansion. The filling of osteochondral samples did not differ from healthy control cartilage $(p=0.085)$ whereas the filling of the chondral samples differed from the controls $(p<0.001)$.

More than half of the osteochondral lesions in each diameter category showed repair tissue with good Safranin-O stain uptake whereas only one of the chondral lesions showed SafraninO positive tissue at the repair site (Table 2). As the repair tissue was absent from two $4 \mathrm{~mm}$ chondral lesion and four $8 \mathrm{~mm}$ chondral lesion samples, those samples were perceived 
negative for Safranin-O uptake. Typically, the osteochondral samples showed hyaline-like

301 cartilage in the deep or middle part of the repair tissue and fibrous tissue on the surface. The

302 best and the worst repairs in each group are shown in Figure 8.

303 Typically, osteochondral defects showed lower values of OARSI score than chondral defects

304 (Table 1). This is indicative of better tissue quality in the osteochondral samples. Loss of

305 Safranin-O uptake was common in all of the defect sizes. No degenerative changes were

306 detected in the control cartilage adjacent to the lesions.

\section{$307 \quad$ Immunohistochemistry}

308 Almost all of the $2 \mathrm{~mm}$ osteochondral samples (4 of 5) showed positive type II collagen 309 staining and only one of these samples showed positive type I collagen staining (Figure 7,

310 Table 2). In the $2 \mathrm{~mm}$ chondral samples, positive staining for type II and type I collagen was

311 shown in 1 of 5 and 4 of 5 samples, respectively. Fibrocartilage formation was evident in the

312 larger chondral and osteochondral lesions where a mixture of type I and type II collagen

313 positive tissue was present. Since the repair tissue was detached from two of the $4 \mathrm{~mm}$

314 chondral lesions and four of the $8 \mathrm{~mm}$ chondral lesions, these were perceived negative for

315 both type I and II collagen.

\section{Discussion}

318 The purpose of this study was to determine the intrinsic repair capacity of equine carpal 319 articular cartilage to set a benchmark for studies evaluating articular cartilage repair strategies

320 using the equine carpus as a model. Knowledge on spontaneous repair capacity and critical 
321 lesion size improves cost-effectiveness and minimizes animal suffering in animal

322 experiments. The quality and quantity of the repair tissue in both chondral and osteochondral

323 defects were evaluated in this study. Complete tissue regeneration was not achieved as the

324 repair tissue structure differed from healthy cartilage in all the defects. Only the osteochondral

325 lesions with $2 \mathrm{~mm}$ diameter showed good Safranin-O staining indicating a good quality of the

326 repair tissue, while equally sized chondral defects failed to spontaneously repair to hyaline

327 cartilage. Chondral defects and osteochondral defects with the diameter of $4 \mathrm{~mm}$ and $8 \mathrm{~mm}$

328 showed depletion of proteoglycans and structural disorganization.

329 The healing of equine carpal cartilage defects was first described by Riddle (17) who created

330 superficial and full-thickness defects in the carpus of four horses $\left(150 \mathrm{~mm}^{2}\right)$ and six ponies

$331\left(100 \mathrm{~mm}^{2}\right)$. He concluded that the superficial defects did not heal past the 8-month time point

332 and that in order for the defects to heal, they should reach the subchondral bone. The

333 importance of the connection to the bone marrow spaces has since been confirmed by others.

$334(18,19)$ Mean filling of both untreated and microfracture-treated chondral defects of $100 \mathrm{~mm}^{2}$

335 in equine carpus in the study by Frisbie was, however, only 65\% or less. (18)

336 In a study evaluating spontaneous healing of full-thickness cartilage defects in the equine

337 carpus by Hurtig et al., (5) lesions with a surface area of $5 \mathrm{~mm}^{2}$ were filled with

338 fibrocartilaginous repair tissue but lesions of $15 \mathrm{~mm}^{2}$ deteriorated to dense fibrous tissue. This

339 is corroborated by our study, where nearly all full-thickness chondral defects of $3 \mathrm{~mm}^{2}(2 \mathrm{~mm}$

340 in diameter) showed fibrocartilaginous repair, and larger defects presented with incomplete

341 fibrocartilage covering or no repair tissue at all. 
342 Spontaneous defect healing reported in previous equine studies is mainly described as filling 343 of the lesions or formation of fibrous tissue and fibrocartilage. $(5,20)$ Fibrocartilage, however, 344 has lower mechanical strength than hyaline cartilage and as such, it is more prone to wearing 345 out. (21) Durable, long-lasting results can only be achieved by restoration of fully functional 346 hyaline cartilage. (6) The focus of interventions aiming at cartilage repair has shifted from 347 simply filling the lesions to restoring mature hyaline cartilage. In order to reliably determine 348 the critical lesion size, it is paramount to evaluate both the quantity and quality of repair 349 tissue. In the present study, only the osteochondral defects showed hyaline-like repair tissue 350 with higher proteoglycan content and better filling after a 12-month follow-up (Figure 4, 351 Figure 8). Even though small full-thickness chondral defects have been thought to heal 352 spontaneously $(3,5,8)$, the results of this study suggest otherwise. Although the filling in the 2 $353 \mathrm{~mm}$ defects was good macroscopically, depletion of proteoglycans was evident both in 354 Safranin-O staining and gadolinium-enhanced MR imaging $\left(T_{1 G d}\right)$. Structural disorganization 355 and fibrocartilage formation were seen in polarized light microscopy and as a mixture of type 356 I and II collagen staining in immunohistochemistry, and low $T_{2}$ and $T_{1 G d}$ values in MRI. $357(22,23)$ The deep part of the repair tissue in the osteochondral defects showed a structure 358 closely resembling that of the healthy control tissue in polarized light microscopy, whereas 359 the chondral defects showed poorly organized tissue in each layer, implying a mechanically 360 weaker tissue structure. These findings substantiate the previous studies that show that the 361 repair tissue originates from the bone marrow (24) and explain the poor outcome of the 362 chondral defects.

363 Although the osteochondral defects showed better repair than chondral defects, the bone voids 364 of the deep osteochondral defects did not heal or even became larger, extending up to $9 \mathrm{~mm}$ 
into the bone, during the 12-month follow-up. Even the smallest $2 \mathrm{~mm}$ osteochondral lesions

366 showed bone pathologies at the time of the post mortem analysis ( 4 of 5 specimens). Frequent

367 cyst formation after a disruption of subchondral bone has been reported in previous equine

368 studies. $(18,25)$ The present study makes no exception: chondral lesions presented with no

369 cysts whereas bone defects were detected in osteochondral lesions of all sizes (2, 4 and 8

$370 \mathrm{~mm})$.

371 The long-term follow-up time of 12 months in this study gives a better understanding of the

372 spontaneous repair capacity of equine carpal joint cartilage than the shorter time periods of

373 previous studies on spontaneous repair. $(8,26)$ Additionally, this study has several

374 methodological benefits, as current state-of-art methods were used in assessing the repair

375 tissue quality. The tissue was evaluated prior to any processing macroscopically and with

$376 \mu \mathrm{CT}$ and MRI. The outcome of polarized light microscopy reflects the mechanical strength of

377 the repair tissue. $(27,28)$ Finally, the overall quality of the repair tissue was assessed with

378 histological and immunohistochemical techniques. The findings of different methods support

379 each other.

380 There were some limitations in this study. Since defects were created in different sites of the

381 joint, they were subjected to different weight-bearing conditions. $(7,8)$ All defects with the

382 same diameter were, however, located on the same site and thus the comparison between

383 chondral and osteochondral defects is justified. The third carpal bone, where the $4 \mathrm{~mm}$ and 8

$384 \mathrm{~mm}$ defects were located, bears most weight and is the site in the equine carpus that is most

385 frequently affected by cartilage pathologies. (29) Nonetheless, not even the $2 \mathrm{~mm}$ lesions

386 located on the less weight-bearing second carpal bone healed well. 
387 Altogether four defects were created in the middle carpal joint of the horses. The combined 388 area of these defects was $94 \mathrm{~mm}^{2}$, which might possibly have affected the repair of the 389 individual lesions, although degenerative changes were absent around the lesions or on the 390 articulating surfaces. Further, it is not uncommon to create more defects per joint when using 391 the equine model $(30,31)$. In our study in the carpus, none of the lesions with a diameter of 4 $392 \mathrm{~mm}\left(13 \mathrm{~mm}^{2}\right)$ or $8 \mathrm{~mm}\left(50 \mathrm{~mm}^{2}\right)$ healed with mature hyaline cartilage. Even the smallest 2 $393 \mathrm{~mm}$ in diameter $\left(3 \mathrm{~mm}^{2}\right)$ lesions, which were initially thought to serve as the control lesions 394 with good spontaneous healing showed repair tissue of questionable quality at 12 months.

\section{Conclusion}

397 The horse is a good animal model for cartilage research and, like humans, it has a very limited 398 spontaneous healing capacity. Based on this study, we recommend using $4 \mathrm{~mm}$ diameter as 399 the critical size for osteochondral lesions and $2 \mathrm{~mm}$ diameter lesion as the critical size for 400 chondral lesions in articular cartilage repair research using the equine carpal joint model. 


\section{Declaration of Interests}

403 The authors report no conflict of interest. The authors alone are responsible for the content 404 and writing of the article.

405

406 Funding

407 This work was supported by the Academy of Finland (Grant \#285909) and the Finnish

408 Funding Agency for Innovation Tekes (Grant 3344/31/03).

409

410 Acknowledgements

411 The authors wish to thank Outi Kiekara (Department of Anatomy, University of Eastern

412 Finland, Kuopio, Finland) for $\mu \mathrm{CT}$ and MR imaging. We thank Nora Rauhala (Department of 413 Applied Physics, University of Eastern Finland, Kuopio, Finland) for conducting the ROI 414 analyses on the MRI data and Eija Rahunen (Department of Anatomy, University of Eastern 415 Finland, Kuopio, Finland) for technical assistance with histological sample preparation. The 416 Biomedicum Imaging Unit (Faculty of Medicine, University of Helsinki) is acknowledged for 417 microscopy services and Hannu Kautiainen (Medcare Oy, Äänekoski, Finland) for the 418 statistical analyses. 


\section{REFERENCES}

(1) Ahern BJ, Parvizi J, Boston R, Schaer TP. Preclinical animal models in single site cartilage defect testing: a systematic review. Osteoarthritis Cartilage 2009 Jun;17(6):705-713.

(2) Moran CJ, Ramesh A, Brama PA, O'Byrne JM, O'Brien FJ, Levingstone TJ. The benefits and limitations of animal models for translational research in cartilage repair. J Exp Orthop 2016 Dec;3(1):1-015-0037-x. Epub 2016 Jan 6.

(3) Chu CR, Szczodry M, Bruno S. Animal models for cartilage regeneration and repair. Tissue Eng Part B Rev 2010 Feb;16(1):105-115.

(4) Malda J, Benders KE, Klein TJ, de Grauw JC, Kik MJ, Hutmacher DW, et al. Comparative study of depth-dependent characteristics of equine and human osteochondral tissue from the medial and lateral femoral condyles. Osteoarthritis Cartilage 2012 Oct;20(10):1147-1151.

(5) Hurtig MB, Fretz PB, Doige CE, Schnurr DL. Effects of lesion size and location on equine articular cartilage repair. Can J Vet Res 1988 Jan;52(1):137-146.

(6) Bernhard JC, Vunjak-Novakovic G. Should we use cells, biomaterials, or tissue engineering for cartilage regeneration? Stem Cell Res Ther 2016 Apr 18;7(1):56-016-0314-3.

(7) Convery FR, Akeson WH, Keown GH. The repair of large osteochondral defects. An experimental study in horses. Clin Orthop Relat Res 1972 Jan-Feb;82:253-262.

(8) McIlwraith CW, Fortier LA, Frisbie DD, Nixon AJ. Equine Models of Articular Cartilage Repair. Cartilage 2011 Oct;2(4):317-326.

(9) McIlwraith CW, Frisbie DD, Kawcak CE. The horse as a model of naturally occurring osteoarthritis. Bone Joint Res 2012 Nov 1;1(11):297-309.

(10) Viren T, Huang YP, Saarakkala S, Pulkkinen H, Tiitu V, Linjama A, et al. Comparison of ultrasound and optical coherence tomography techniques for evaluation of integrity of spontaneously repaired horse cartilage. J Med Eng Technol 2012 Apr;36(3):185-192.

(11) Kulmala KA, Pulkkinen HJ, Rieppo L, Tiitu V, Kiviranta I, Brunott A, et al. Contrast-Enhanced Micro-Computed Tomography in Evaluation of Spontaneous Repair of Equine Cartilage. Cartilage $2012 \mathrm{Jul} ; 3(3): 235-244$.

(12) Rautiainen J, Lehto LJ, Tiitu V, Kiekara O, Pulkkinen H, Brunott A, et al. Osteochondral repair: evaluation with sweep imaging with fourier transform in an equine model. Radiology 2013 Oct;269(1):113-121.

(13) Rieppo J, Hallikainen J, Jurvelin JS, Kiviranta I, Helminen HJ, Hyttinen MM. Practical considerations in the use of polarized light microscopy in the analysis of the collagen network in articular cartilage. Microsc Res Tech 2008 Apr;71(4):279-287. 
(14) Muhonen V, Salonius E, Haaparanta AM, Jarvinen E, Paatela T, Meller A, et al. Articular cartilage repair with recombinant human type II collagen/polylactide scaffold in a preliminary porcine study. J Orthop Res 2015 Nov 17.

(15) Schindelin J, Arganda-Carreras I, Frise E, Kaynig V, Longair M, Pietzsch T, et al. Fiji: an opensource platform for biological-image analysis. Nat Methods 2012 Jun 28;9(7):676-682.

(16) McIlwraith CW, Frisbie DD, Kawcak CE, Fuller CJ, Hurtig M, Cruz A. The OARSI histopathology initiative - recommendations for histological assessments of osteoarthritis in the horse. Osteoarthritis Cartilage 2010 Oct;18 Suppl 3:S93-105.

(17) Riddle WE,Jr. Healing of articular cartilage in the horse. J Am Vet Med Assoc 1970 Dec 1;157(11):1471-1479.

(18) Frisbie DD, Trotter GW, Powers BE, Rodkey WG, Steadman JR, Howard RD, et al. Arthroscopic subchondral bone plate microfracture technique augments healing of large chondral defects in the radial carpal bone and medial femoral condyle of horses. Vet Surg 1999 Jul-Aug;28(4):242-255.

(19) Vachon A, Bramlage LR, Gabel AA, Weisbrode S. Evaluation of the repair process of cartilage defects of the equine third carpal bone with and without subchondral bone perforation. Am J Vet Res 1986 Dec;47(12):2637-2645.

(20) Vachon AM, McIlwraith CW, Trotter GW, Norrdin RW, Powers BE. Morphologic study of repair of induced osteochondral defects of the distal portion of the radial carpal bone in horses by use of glued periosteal autografts [corrected. Am J Vet Res 1991 Feb;52(2):317-327.

(21) Hunziker EB. The elusive path to cartilage regeneration. Adv Mater 2009 Sep 4;21(32-33):34193424.

(22) Nieminen MT, Nissi MJ, Mattila L, Kiviranta I. Evaluation of chondral repair using quantitative MRI. J Magn Reson Imaging 2012 Dec;36(6):1287-1299.

(23) Nissi MJ, Toyras J, Laasanen MS, Rieppo J, Saarakkala S, Lappalainen R, et al. Proteoglycan and collagen sensitive MRI evaluation of normal and degenerated articular cartilage. J Orthop Res 2004 May;22(3):557-564.

(24) Shapiro F, Koide S, Glimcher MJ. Cell origin and differentiation in the repair of full-thickness defects of articular cartilage. J Bone Joint Surg Am 1993 Apr;75(4):532-553.

(25) Kold SE, Hickman J, Melsen F. An experimental study of the healing process of equine chondral and osteochondral defects. Equine Vet J 1986 Jan;18(1):18-24.

(26) Frisbie DD, Lu Y, Kawcak CE, DiCarlo EF, Binette F, McIlwraith CW. In vivo evaluation of autologous cartilage fragment-loaded scaffolds implanted into equine articular defects and compared with autologous chondrocyte implantation. Am J Sports Med 2009 Nov;37 Suppl 1:71S-80S.

(27) Vasara AI, Hyttinen MM, Lammi MJ, Lammi PE, Langsjo TK, Lindahl A, et al. Subchondral bone reaction associated with chondral defect and attempted cartilage repair in goats. Calcif Tissue Int 2004 Jan;74(1):107-114. 
(28) Julkunen P, Harjula T, Iivarinen J, Marjanen J, Seppanen K, Narhi T, et al. Biomechanical, biochemical and structural correlations in immature and mature rabbit articular cartilage. Osteoarthritis Cartilage 2009 Dec;17(12):1628-1638.

(29) Palmer JL, Bertone AL, Litsky AS. Contact area and pressure distribution changes of the equine third carpal bone during loading. Equine Vet J 1994 May;26(3):197-202.

(30) Hurtig M, Pearce S, Warren S, Kalra M, Miniaci A. Arthroscopic mosaic arthroplasty in the equine third carpal bone. Vet Surg 2001 May-Jun;30(3):228-239.

(31) Nixon AJ, Rickey E, Butler TJ, Scimeca MS, Moran N, Matthews GL. A chondrocyte infiltrated collagen type I/III membrane (MACI(R) implant) improves cartilage healing in the equine patellofemoral joint model. Osteoarthritis Cartilage 2015 Apr;23(4):648-660. 
501 Figure 1. Schematic drawing of the left equine carpal bones II-IV with the four different 502 lesion sizes marked with dashed lines.

503 Figure 2. Representative MRI relaxation time maps of the chondral (top row) and osteochondral (bottom row) lesions of $2 \mathrm{~mm}$ diameter. Lesion site is immediately below the scale bar $(2 \mathrm{~mm})$. Shorter $T_{1 \mathrm{Gd}}$ relaxation times were observed in both lesions, but more prominently so in the chondral lesion (a). Slight differences as compared to the adjacent tissue were also evident in the $T_{2}$ and $T_{1}$ relaxation time maps $(\mathrm{a}, \mathrm{b})$. The ROI for control tissue is exemplified in the magnification of $b$. In the magnification of $c$, the ROI 1 for the repair tissue is marked with red, and the ROI 2 aligned to adjacent healthy cartilage is marked with green.

Figure 3. Photographs of the joints taken directly after sacrificing the animals. Chondral lesions are in the left column and osteochondral in the right column. Each horse is represented in its own row. Macroscopically, 5 of 5 osteochondral and 3 of 5 chondral lesions with the diameter of $2 \mathrm{~mm}$ were filled with repair tissue. Of the $4 \mathrm{~mm}$ lesions, only one chondral lesion (horse D) was well filled, the other lesions were easily distinguishable and not filled to the level of the surrounding cartilage. Each $8 \mathrm{~mm}$ defect was clearly visible but one osteochondral defect (horse E) showed good filling in the middle of the lesion.

Figure 4. Micro-computational tomographic image of the middle part of each specimen. The osteochondral lesions (b, d, f) presented with subchondral bone resorption, whereas the bone structure in the chondral lesions (a, c, e) was either undisturbed or slightly decreased in density. Arrowheads show the site of the original defect.

Figure 5. Mean relaxation time values (ms) of the magnetic resonance imaging in the different cartilage ROIs. Results for the repair tissue only (ROI 1) in each group are shown in (a), and the area aligned to the adjacent healthy cartilage (ROI 2) was divided into a superficial half (b) and deep half (c). Chondral lesions are colored white and osteochondral ones dark gray. The whiskers represent $95 \%$ confidence interval. Statistically significant $p$ values are marked in the images.

Figure 6. Bar diagrams showing the mean parallelism index (PI, top row) of the collagen fibrils and the mean orientation (bottom row) of the fibrils. Chondral lesions are colored white and osteochondral lesions dark gray. The whiskers represent $95 \%$ confidence interval. Statistically significant $p$ values are marked in the images.

Figure 7. Representative osteochondral defect with a diameter of $2 \mathrm{~mm}$. a) Polarized light microscopy showed the change in collagen orientation toward the typical tangential orientation in the superficial layer. b) Safranin-O staining showed good filling and abundant proteoglycans in the deep part of the repaired cartilage tissue. c) Immunohistochemical staining for type II collagen. Scale bar: $500 \mu \mathrm{m}$.

Figure 8. The best and worst Safranin-O stained histological section in each study group. Repair tissue seemed to originate partly from the subchondral bone at the sites where the calcified cartilage was disrupted (arrows). Scale bars: $1 \mathrm{~mm}$. 